\title{
RADIATION CHARACTERISTICS OF ULTRASONIC TIP ECHO AND ITS APPLICATION TO DETECT FATIGUE CRACK CLOSURE IN WELDING RESIDUAL STRESS FIELDS
}

\author{
Chitoshi MIKI ${ }^{1}$, Ryohei ONISHI ${ }^{2}$, Takumi KUBOTA ${ }^{3}$ and Takeshi HANJI ${ }^{4}$ \\ ${ }^{1}$ Fellow of JSCE, Professor, Dept. of Civil Eng., Tokyo Institute of Technology \\ (2-12-1 Ookayama, Meguro-ku, Tokyo 152-8552, Japan) \\ E-mail: miki@cv.titech.ac.jp \\ ${ }^{2}$ Student Member of JSCE, Graduate Student, Dept. of Civil Eng., Tokyo Institute of Technology \\ (2-12-1 Ookayama, Meguro-ku, Tokyo 152-8552, Japan) \\ E-mail: ohishi.r.aa@m.titech.ac.jp \\ ${ }^{3}$ Student Member of JSCE, Graduate Student, Dept. of Civil Eng., Tokyo Institute of Technology \\ (2-12-1 Ookayama, Meguro-ku, Tokyo 152-8552, Japan) \\ E-mail: kubota.t.aa@m.titech.ac.jp \\ ${ }^{4}$ Member of JSCE, Postdoctoral Fellow, Center for Urban Earthquake Eng., Tokyo Institute of Technology \\ (2-12-1 Ookayama, Meguro-ku, Tokyo 152-8552, Japan) \\ E-mail: hanji@cv.titech.ac.jp
}

\begin{abstract}
Ultrasonic tip echo is scattered wave which radiates all direction from the sharp tip of reflection source such as fatigue cracks. Tip echo has been utilized for sizing defects as so-called "tip echo method" and also may apply to identify fatigue cracks from other defects. In this study, radiation characteristics of the tip echo were experimentally examined. A linear phased array ultrasonic testing system was applied to send and receive ultrasonic waves in various directions. The experimental results demonstrated the relationship between incident wave directions and directivity of the tip echo. Besides, the tip echo method was applied to a fatigue crack in a welded steel plate, and damping of echoes due to fatigue crack closure was indicated.
\end{abstract}

Key Words : ultrasonic tip echo, radiation characteristics, fatigue crack tip closure, residual stress

\section{INTRODUCTION}

One of the most critical pieces of information obtained during non-destructive testing (NDT) of structural components suspected of containing fatigue cracks, and vital to further decisions concerning preventive measures, is whether a detected flaw is actually a fatigue crack or a flaw left during the manufacturing process, such as a weld defect. Preventive measures are necessary if the flaw is a fatigue crack, but immediate measures are not needed if it proves to be a flaw remaining after manufacturing. One common NDT method used to characterize flaws and identify fatigue cracks is detecting the fluctuations of ultrasonic echoes due to crack tip closure under stress fluctuations ${ }^{1}$.

Ultrasonic crack tip echoes are scattered echoes generated when a sharply defined tip of a crack is exposed to ultrasonic waves (see Fig.1(a)). One of the essential characteristics of these echoes is that they are radiated in all directions, irrespective of the orientation of the incident ultrasonic signal. According to this characteristic of tip echoes, even the standard method, using a single probe, can receive the tip echo, and it has been employed to measure the length of flaws, as so-called "tip echo method"2)-8). However, no echoes can be detected when ultrasonic waves strike a crack wall, because the waves striking the crack wall will not always returns to the probe (see Fig.1(b)). Therefore, in order to confirm that the flaw is a fatigue crack, the user should check that the returning signal is actually from the sharp tip of a crack and that the echoes fluctuate in concert with stress fluctuations.

Ultrasonic probes show high directionality in both transmission and reception of echoes. As such, crack tip echoes have been used to estimate the dimensions of cracks, but the location of the probe yielding the most efficient detection and the properties of crack 


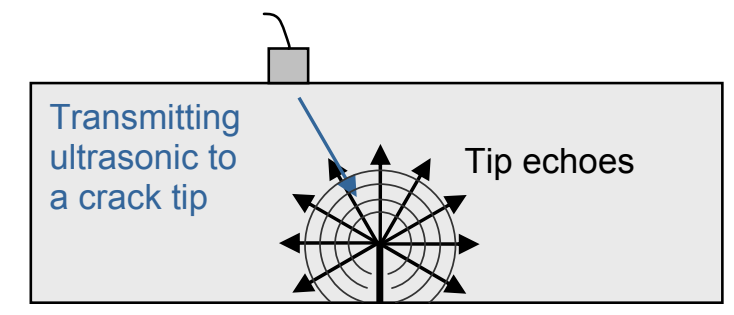

(a) Transmitting ultrasonic to a crack tip

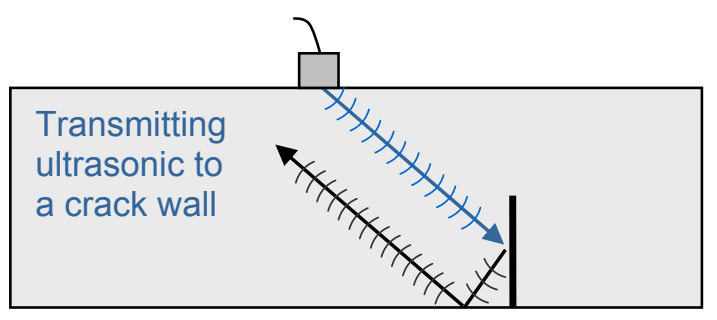

(b) Transmitting ultrasonic to a crack wall surface

Fig.1 Reflection wave from crack tip and crack wall.

tip echoes have not been verified yet. In recent years, it has become possible to confer probes with high directionality, in any desired direction, using phased array systems ${ }^{8)-11)}$. The system can show the orientations producing the most efficient tip echo reception, which will become possible to make more accurate observations of crack tip echoes and broaden the usable range for these measurements.

In this study, in order to investigate the radiation characteristics of the tip echo, a linear phased array ultrasonic testing system was applied to transmit waves to the tip of crack with various angles and to receive the tip echo from various directions. Besides, the tip echo method was employed to detect closure and opening behavior of fatigue crack tip existing in a welded steel plate.

\section{REFLECTION CHARACTERISTICS OF CRACK TIP ECHO}

\section{(1) Specimens}

The configurations of the specimens are shown in Fig.2. Compact tension specimen cut out from a steel plate was used in this test. A slit was machined in specimen CT-1 and left as-is, while in specimen CT-2, after machining the slit, the specimen was stressed in order to obtain a fatigue crack from the slit root. The crack length was approximately $10 \mathrm{~mm}$, which was almost equal to the slit size.

\section{(2) Experimental method}

The 64-channel linear phased array probe (Japan Probe, $5 \mathrm{~K} 0.55 \times 1.18 \mathrm{~N}-64 \mathrm{CH} \mathrm{P}=0.59)$, shown in

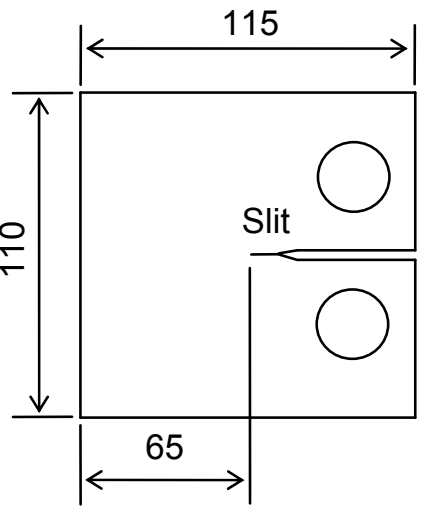

(a) Specimen CT-1

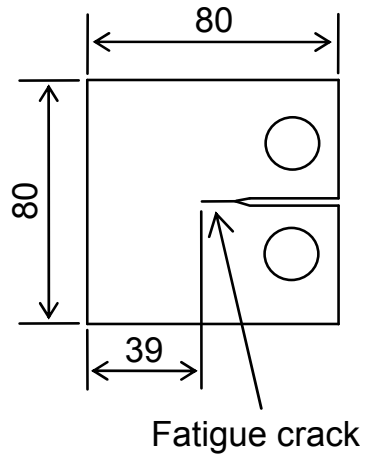

(b) Specimen CT-2
Fig.2 Specimen (unit: $\mathrm{mm}$ ).

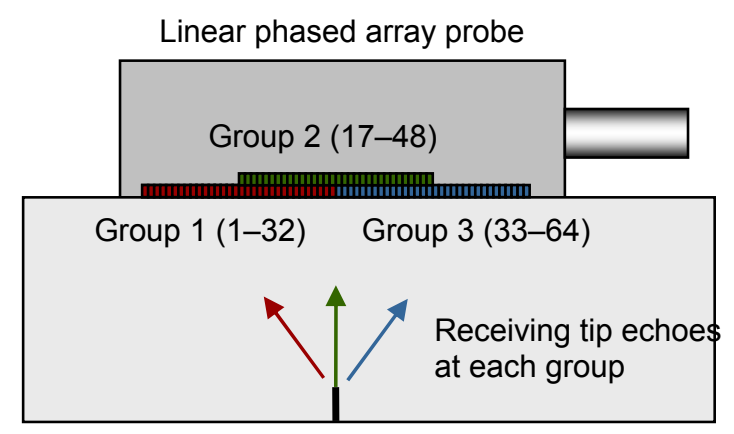

Fig.3 Linear phased array probe.

Fig.3, was used in the present study. The phased array probe consists of many small elements of transducers. By combining many small waves generated from small elements with time delay, the phased array probe can create one incident wave with arbitrary angle. As shown in Fig.4, the incident direction of the wave can be controlled using the steering ability of the phased array, and the incident wave can be concentrated at the tip of the flaw using the array focusing function. With use of the receiving ability of the phased array, the reception angle of the echo can be also controlled. Radiation characteristics of the tip echo were investigated through a combination of these functions. As shown in Fig.3, the front row of 32 oscillators were designated Group 1, oscillators \#17-48 were designated Group 2, \#33-64 were designated Group 3. Each group was used for both transmitting and receiving the waves and for recording of crack tip echoes.

The layout of the experiment is shown in Fig.5. The direction of transmission to and reflection from the crack tip was determined by the angle between a 


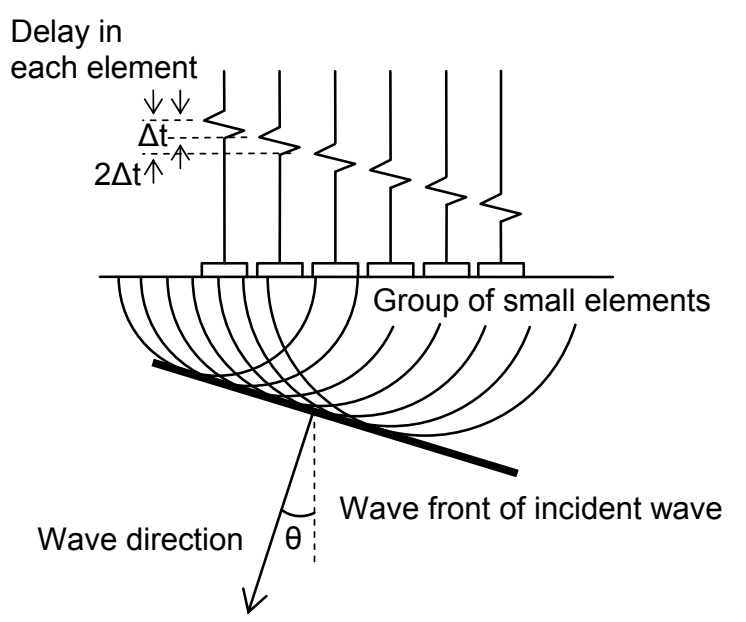

(a) Steering

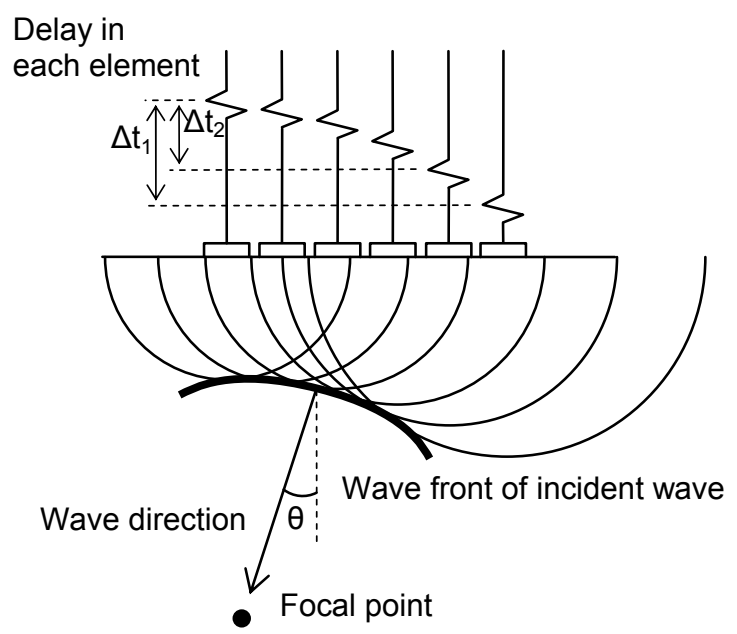

(b) Focusing

Fig.4 Steering and focusing ability of phased array system.

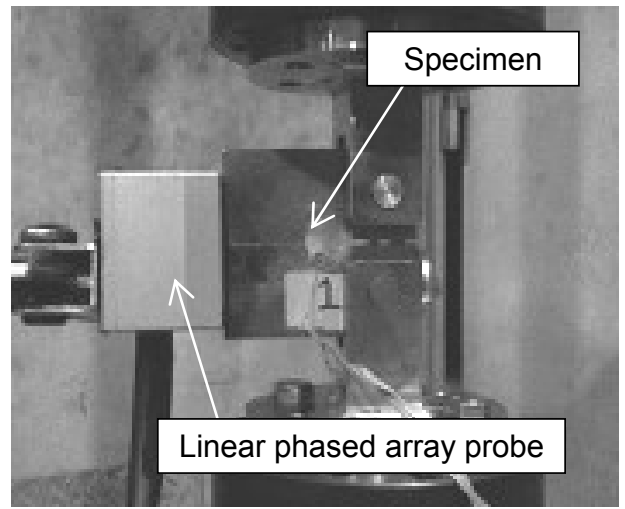

Fig.5 Layout of experiment.

line extended from the slit (or the crack) and a line from the center of the group of oscillators to the target tip (see Fig.6). In this experiment, using both the steering and the focusing ability, ultrasonic waves were transmitted in various angles to focus on the tip. A distribution of the crack tip echoes produced at various incident direction was measured at various receiving direction by varying both the transmission angle and echo scan locations.

\section{(3) Experimental results}

The detected echoes from different receiving angles when transmitting waves to the slit in specimen CT-1 at angles of $8^{\circ}, 16^{\circ}$ and $24^{\circ}$ are shown in Figs.7(a), (b) and (c), respectively. The vertical axes on the graphs represent tip echo level, normalized to the echo height received when the incident and the receiving angle are the same. The reflected echo was strongest when receiving echoes at the same angle as the transmission in all cases. This result indicates that

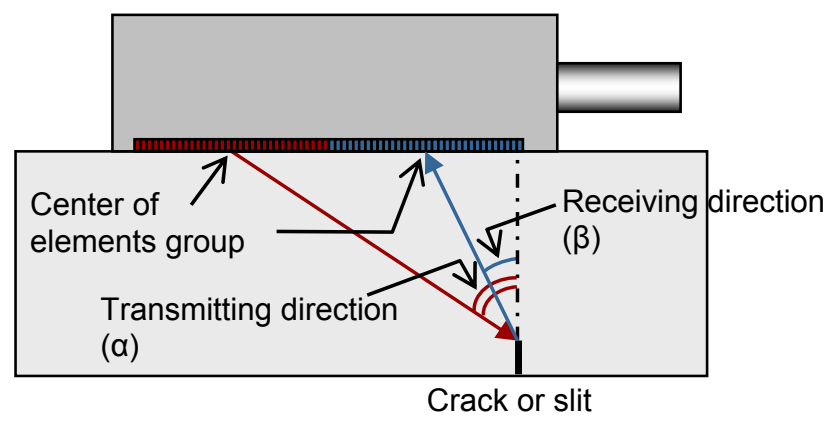

Fig.6 Definition of transmitting and receiving angle.

the tip echoes tend to be reflected strongly back toward the source of the ultrasonic wave.

Fig.8 shows the detected echoes from the different receiving angles in specimen $\mathrm{CT}-2$ when the incident angle to the fatigue crack tip was set to be $13^{\circ}$. It can be observed that the tip echo from the fatigue crack has the same tendency as that from the slit.

The directionality of tip echo intensity when changing the transmitting direction is shown in Fig.9. The height of the tip echo was measured at transmission angles of $-8^{\circ}$ to $24^{\circ}$, in increments of $8^{\circ}$. The receiving angle of the tip echo was set as the same as the incident wave. The tip echo level in the ordinate is normalized to the maximum intensity of the echo received during the measurements. Test results indicate that the strongest echoes were received when the incident wave angle was $0^{\circ}$ (i.e., when the ultrasonic wave was transmitted from directly above the slit), and that the echo magnitude tended to decrease with distance from the slit or the crack. 


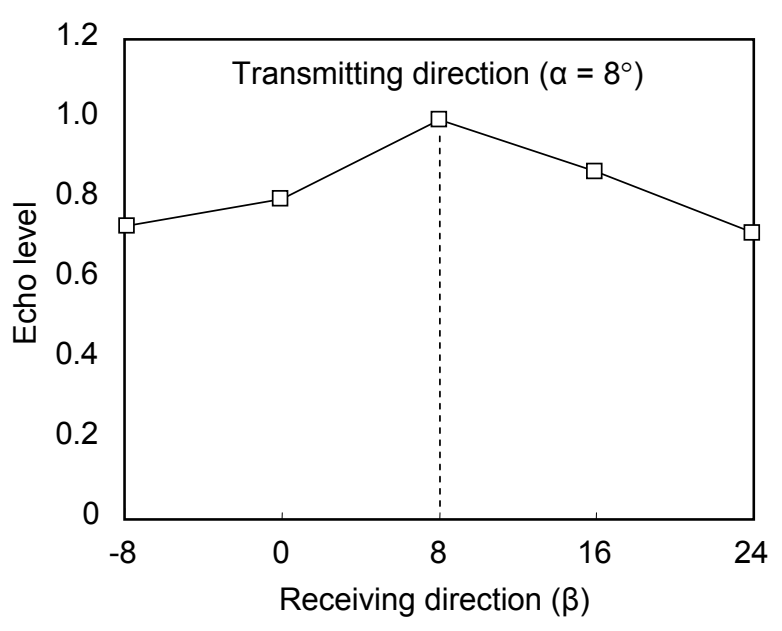

(a) $\alpha=8^{\circ}$

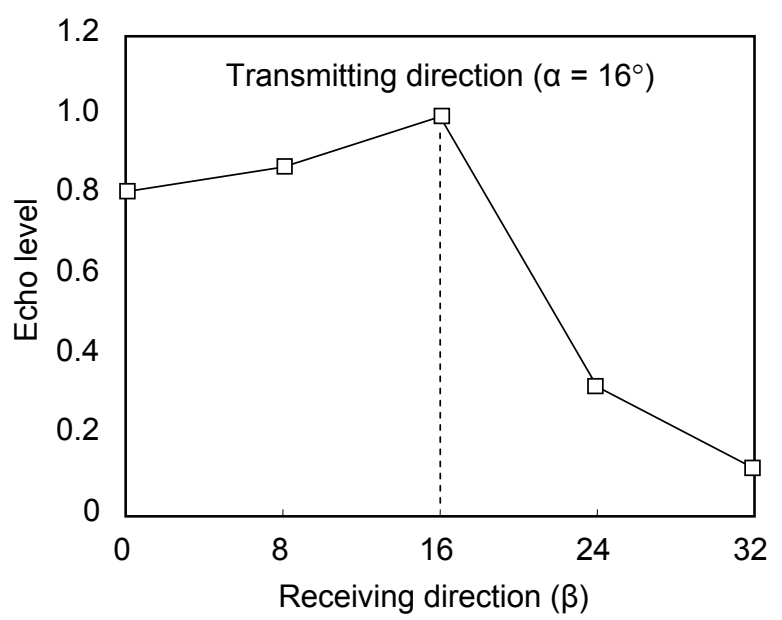

(b) $\alpha=16^{\circ}$

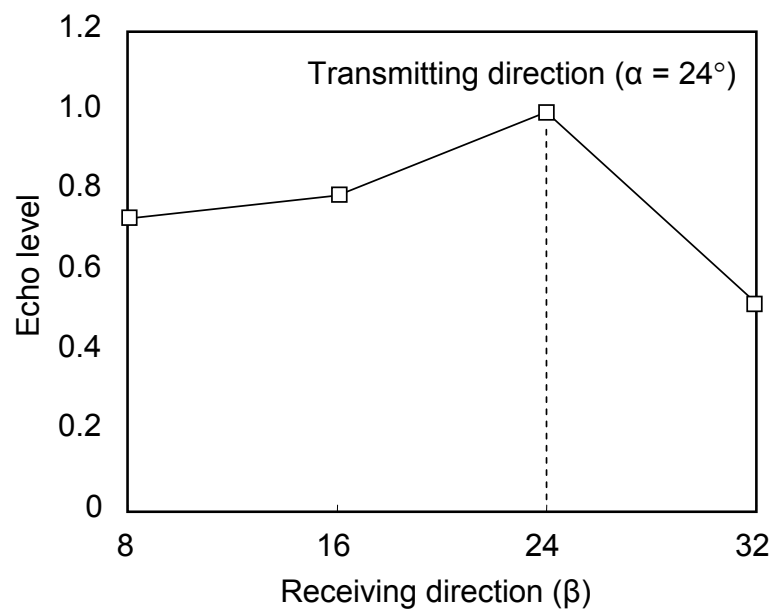

(c) $\alpha=24^{\circ}$

Fig.7 Tip echo distribution (specimen CT-1).

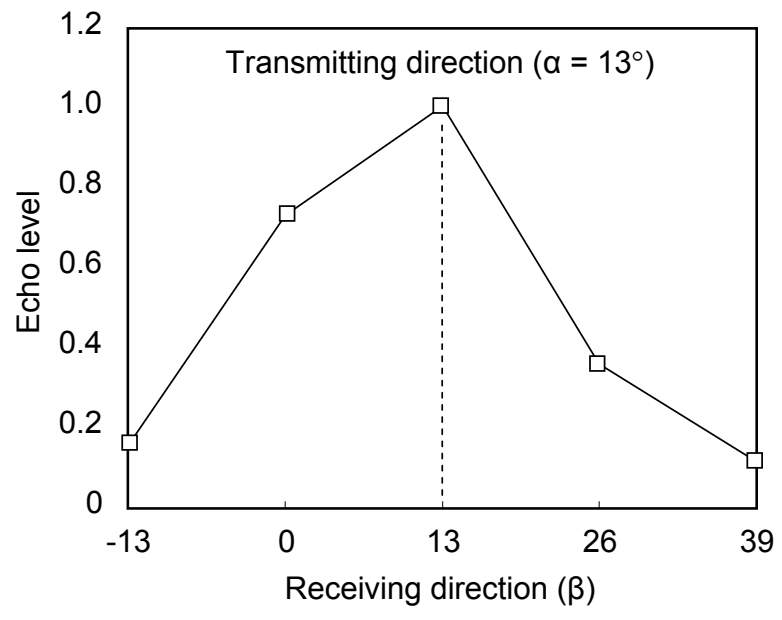

Fig.8 Tip echo distribution in $\alpha=13^{\circ}$ (specimen CT-2).

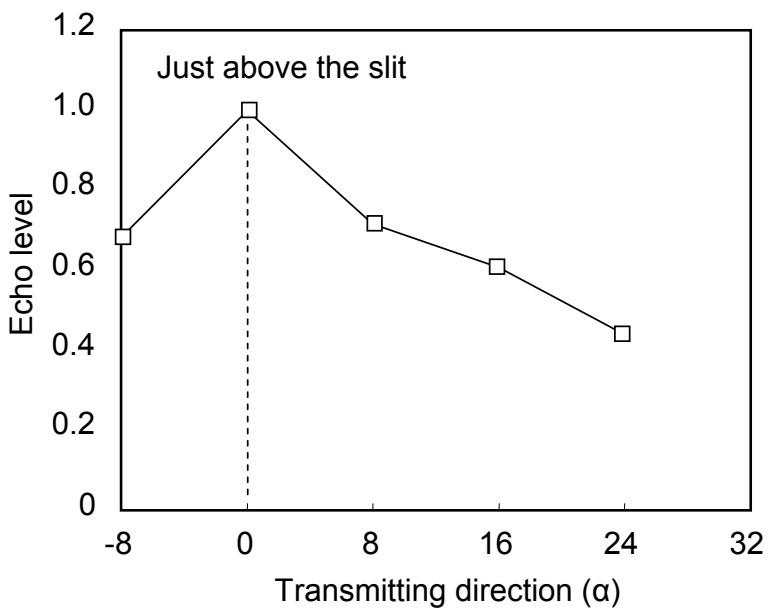

Fig.9 Change of echo level due to transmitting direction (specimen CT-1).

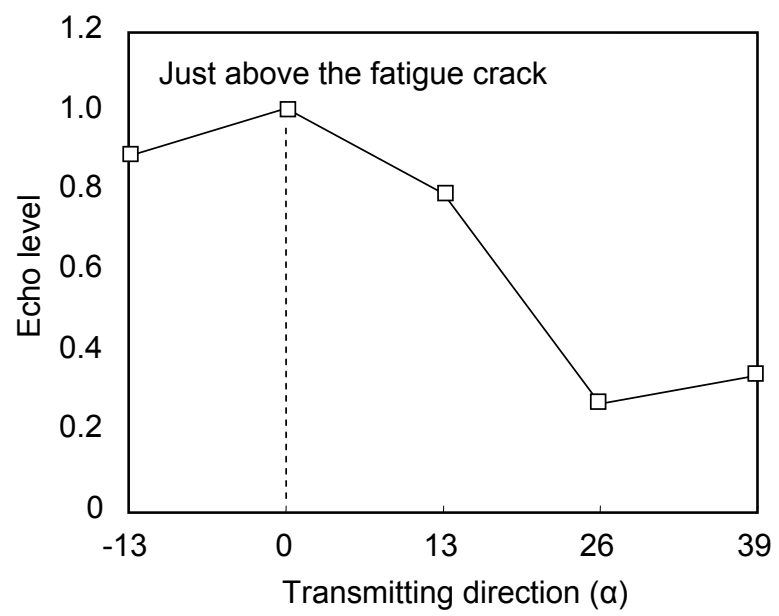

Fig.10 Change of echo level due to transmitting direction (specimen CT-2). 

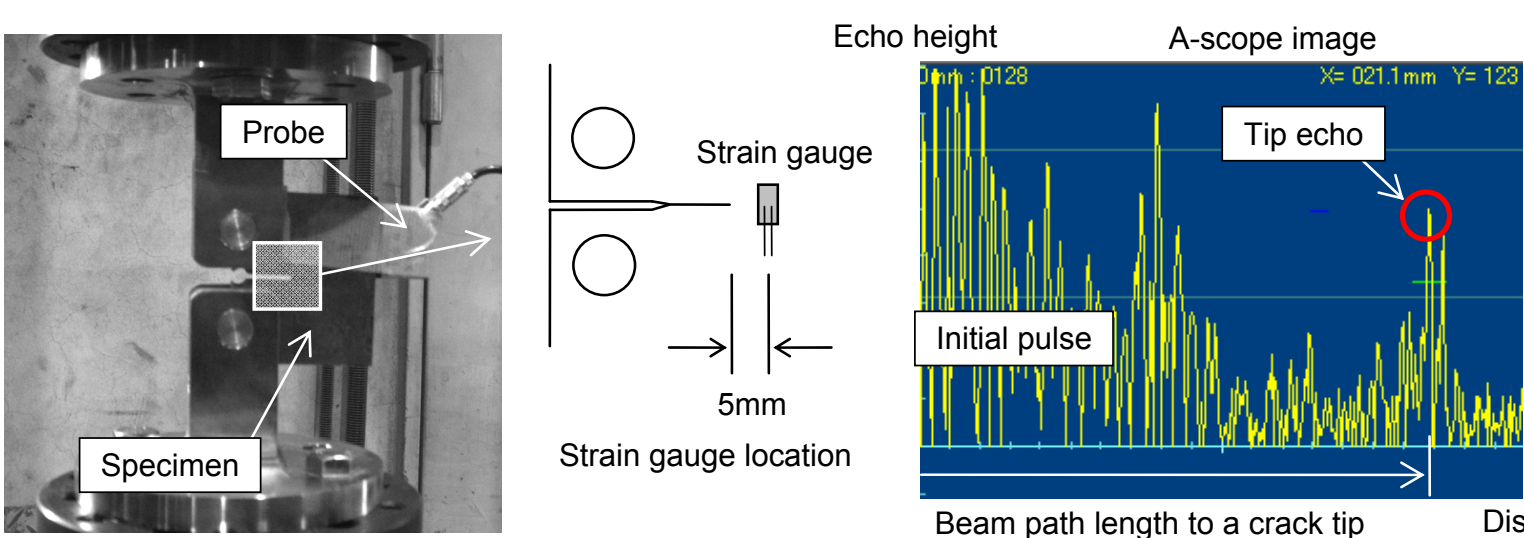

Beam path length to a crack tip

Distance

Fig.11 Test setup and an example of A-scope image.

The intensity of crack tip echoes when ultrasonic waves were transmitted to the fatigue crack tip in specimen CT-2 at angles of $-13^{\circ}$ to $39^{\circ}$, in increments of $13^{\circ}$ is shown in Fig.10. Identical to the results of echoes from the slit in specimen CT-1, the strongest echoes were received when transmission occurred from directly overhead, and the return signals tended to weaken as the incident angle was increased.

As mentioned above, the experimental results make it clear that the tip echo can be received with high intensity when the ultrasonic wave was sent from above the crack tip and the incident and the receiving angle are the same. This supports that a conventional single-type ultrasonic probe, in which the transmitting angle corresponds with the receiving angle, can be sufficiently applied to detect the tip echo. Therefore in the following experiments, a single-type probe was employed which enables a more convenient and simpler measurement.

\section{CHANGES IN TIP ECHOES DUE TO CRACK TIP CLOSURE}

The crack tip closure was measured by applying the tip echo method in specimen CT-2, using a standard probe with $5 \mathrm{MHz}$ in frequency and refraction angle of $45^{\circ}$ (Japan Probe, 5Z10 $\times 10$ A45). Fig.11 shows the test setup, including an A-scope image of the crack tip when the signals were centered on the tip. The leading edges of the echoes are the return signals from the crack tip.

The relationships between the load and the output of a strain gauge attached $5 \mathrm{~mm}$ away from a crack tip (in Fig.11), and between the load and the tip echo level are shown in Fig.12(a). The maximum loading was $5 \mathrm{kN}$. In the figure, the knee points of the rela-

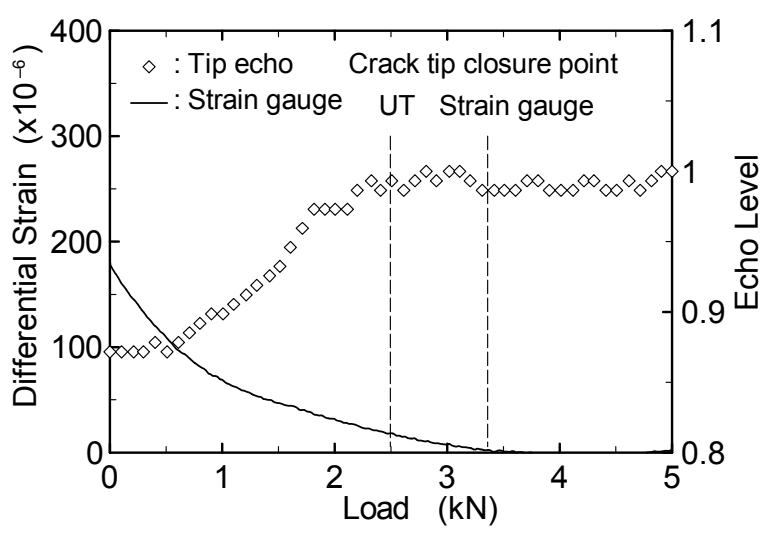

(a) Measurement results

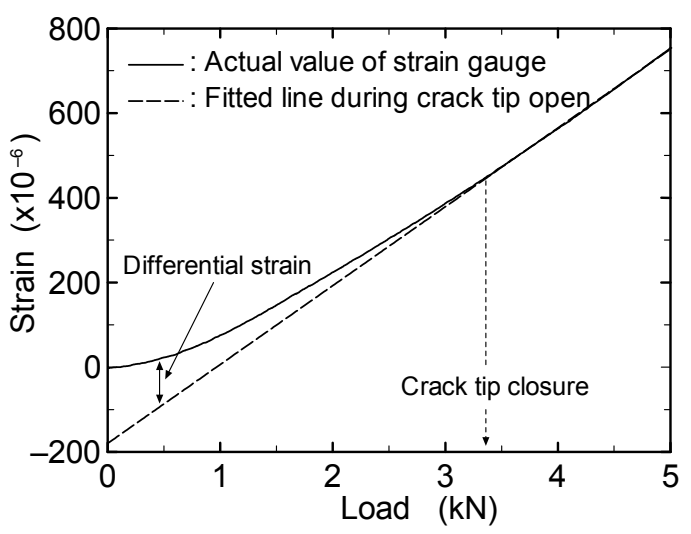

(b) Unloading elastic compliance method

Fig.12 Results of static loading test for specimen CT-2.

tionships can be considered as the fatigue crack tip closure point. The crack tip closure points from the results of strain measurement were determined by applying the unloading elastic compliance method ${ }^{12)}$. In the compliance method, differential strains are calculated from the differences between strain gauge values and a fitted line while the crack tip is open, as 


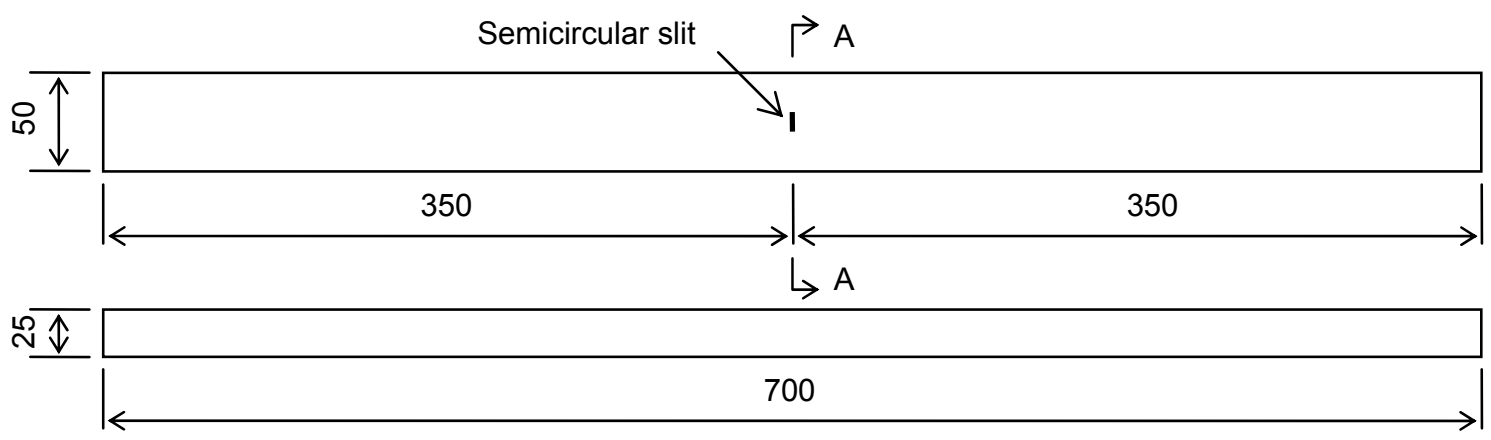

(a) Specimens CC-1

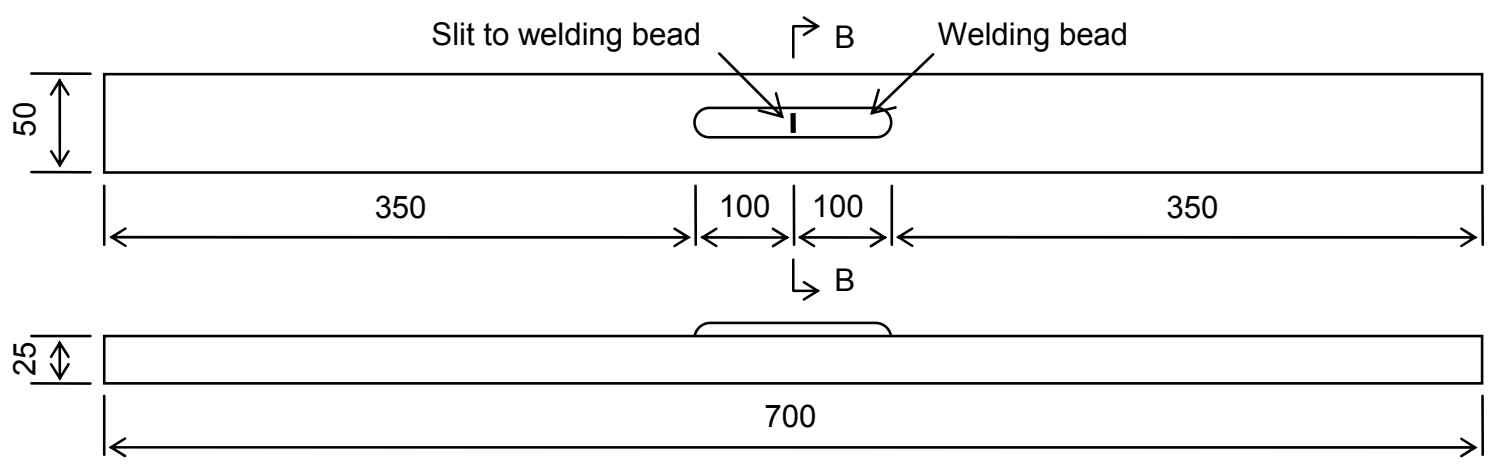

(b) Specimens CC-2
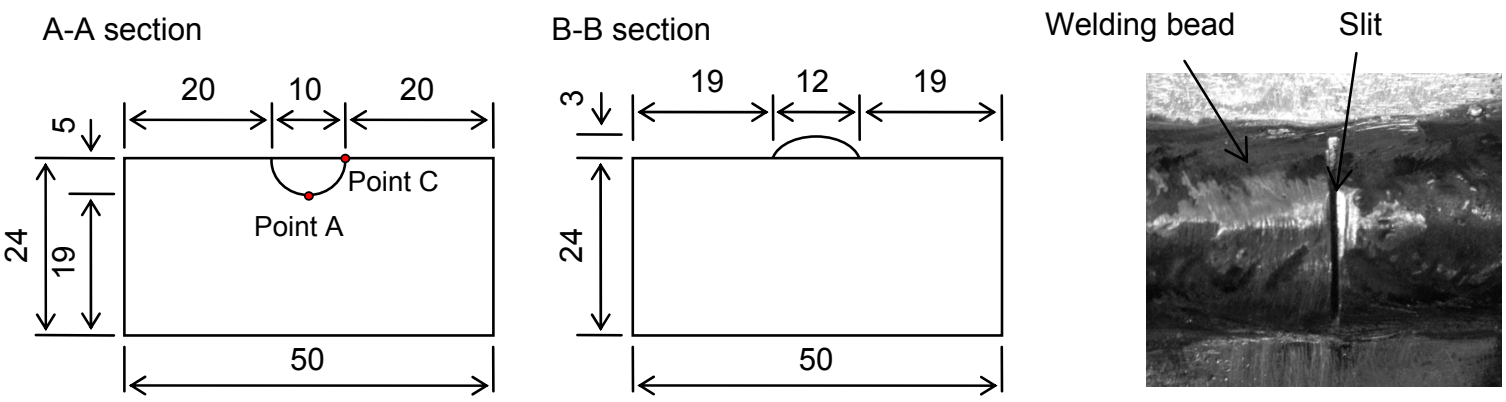

(c) Detail around a slit

Fig.13 Specimens (Unit: mm).

shown in Fig.12(b). The load-differential strain curve shows that the differential strain became 0 around a load of $3.4 \mathrm{kN}$, while the relationship between the load and the echo level, which is normalized to the maximum echo height, shows that the echo level became constant once the load had exceeded about $2.5 \mathrm{kN}$. The differences in the crack closure load between two methods may be caused because the strain gauge detected the crack closure on a plate surface, while the ultrasonic tip echo detected crack behavior of inside a plate. The result verified that both methods can detect the crack closure and that the magnitude of echoes reflected from a fatigue crack fluctuates as the crack opens and closes under stress fluctuations.

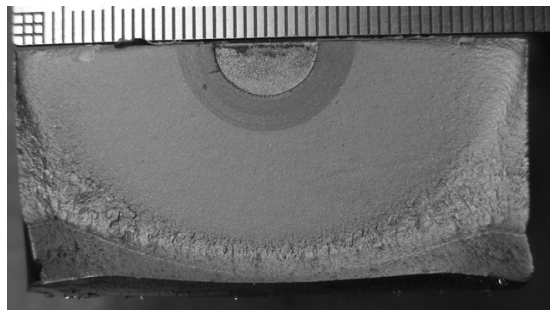

(a) Specimen-CC1

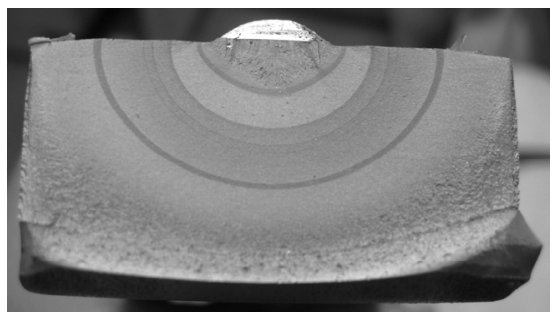

(b) Specimen-CC2

Fig.14 Fracture surfaces. 


\section{APPLICATION OF TIP ECHO METHOD TO CRACKS IN RESIDUAL STRESSES}

The tip echo method was applied to a three dimensional shaped crack existing in a welded steel plate to grasp crack tip closure and opening behavior in the welding residual stress field.

\section{(1) Specimens}

Fig.13 shows the configurations of the specimens. In specimen CC-1, a semicircular slit with $10 \mathrm{~mm}$ diameter was carefully machined at the mid-width of a plate not to generate the residual stress around the slit and left as-is. On the other hand in specimen CC-2, welding bead was put on one side of a plate to produce the residual stress fields, and a slit was machined on the bead. Both specimens were stressed in order to initiate and propagate a fatigue crack from the slit root. During the fatigue test, beach marks shown in Fig.14 were introduced into the specimen to measure the crack depth after the test.

The residual stress distribution in thickness direction about specimen CC-2 was measured by the slitting method and shown in Fig.15. It can be confirmed that high tensile residual stresses were produced around the welding bead.

\section{(2) Experimental method}

The crack closure and opening behavior was detected by a point focusing probe with $5 \mathrm{MHz}$ in frequency, refraction angle of $45^{\circ}$ and focusing range of from $7 \mathrm{~mm}$ to $26 \mathrm{~mm}$ (Japan Probe, 5Z10A45 PF17). Overview of the experiment is illustrated in Fig.16. The probe was set on the opposite side of a cracked surface. While loading and unloading processes, changes in the intensity of the tip echo were determined at different horizontal locations of the probe in the range of $y=-6 \mathrm{~mm}$ to $6 \mathrm{~mm}$ in Fig.16. As a result of measurements, the crack tip closure and opening was detected along the crack front.

\section{(3) Experimental results}

The detected echoes to the semicircular slit in specimen CC-1 at locations of $\mathrm{y}=0 \mathrm{~mm}, 2 \mathrm{~mm}$ and $4 \mathrm{~mm}$ are shown in Fig.17. The ordinate on the graphs represent the echo level normalized to the intensity of the highest tip echo received while the crack tip opens. In the graphs, tip echoes are almost constant regardless of magnitude of load, indicating that the artificial slit remained open at any loads.

The tip echoes to a fatigue crack initiating from the slit in specimen CC-1 detected at locations of $\mathrm{y}=$ $0 \mathrm{~mm}, 2 \mathrm{~mm}$ and $4 \mathrm{~mm}$ are shown in Fig.18. The crack depth was approximately $7.5 \mathrm{~mm}$. At any locations, the crack tip closure and opening can be observed. The crack closure loads, defined as the knee point of the relationship shown in the figure, change along the crack front, which tends to increase as distance from the crack center $(40 \mathrm{kN}, 71 \mathrm{kN}$ and $120 \mathrm{kN}$ at $\mathrm{y}=0 \mathrm{~mm}$, $2 \mathrm{~mm}$ and $4 \mathrm{~mm}$ ). The similar tendencies can be observed for the crack opening load. That is to say, the stress ratio at the crack tip, $\mathrm{U}$, varies along the crack
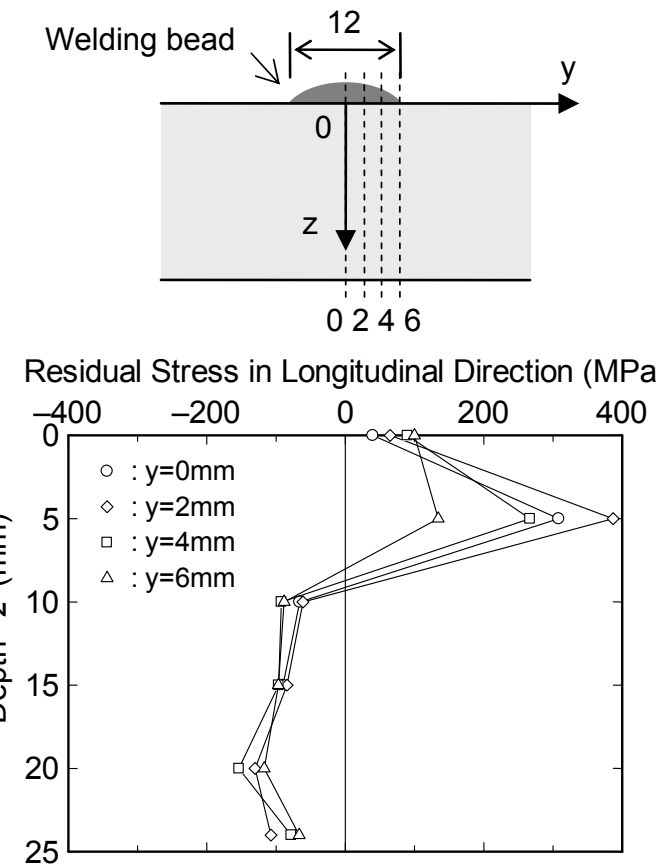

Fig.15 Residual stress distribution in thickness direction for specimen $\mathrm{CC}-2$.

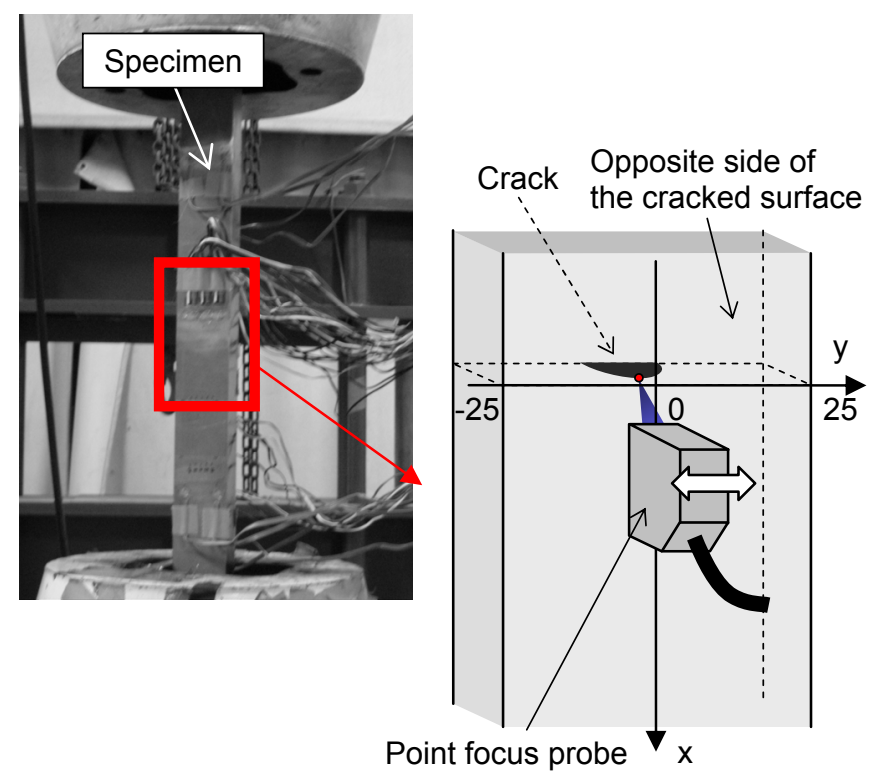

Fig.16 Overview of experiment. 


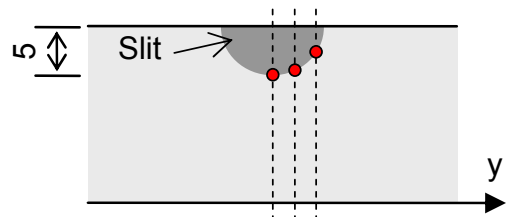

024

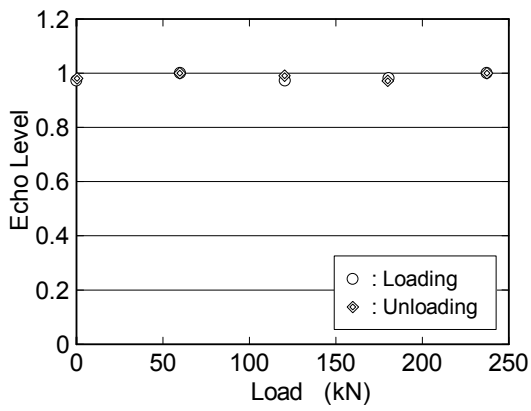

(a) $\mathrm{y}=0 \mathrm{~mm}$

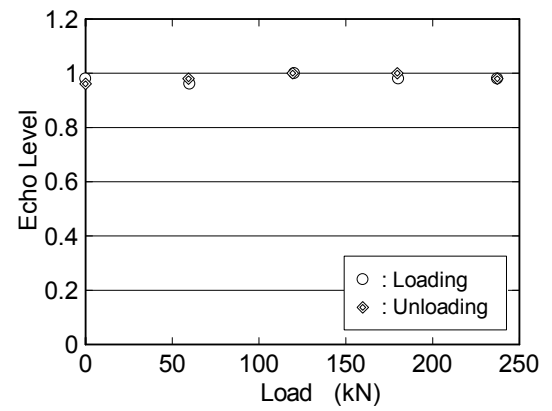

(b) $\mathrm{y}=2 \mathrm{~mm}$

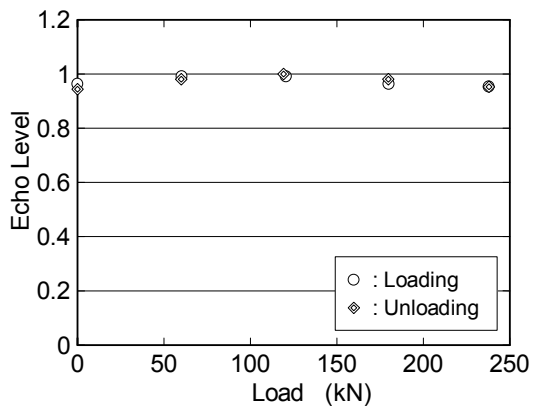

(c) $\mathrm{y}=4 \mathrm{~mm}$

Fig.17 Relationship between load and tip echoes (specimen CC-1 without fatigue crack).

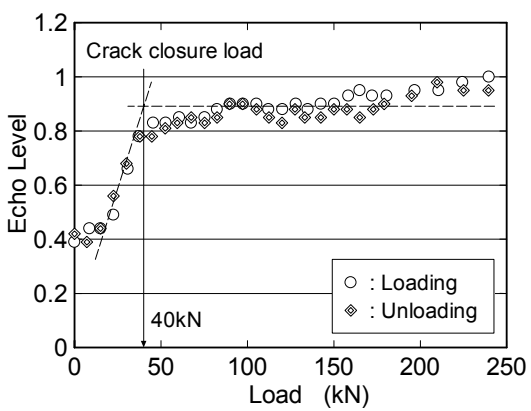

(a) $\mathrm{y}=0 \mathrm{~mm}$

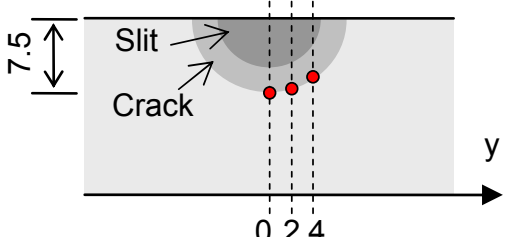

024

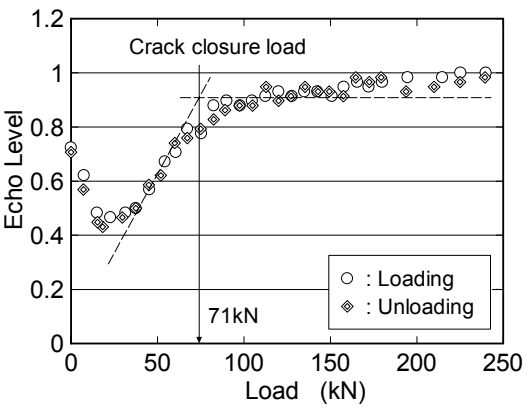

(b) $\mathrm{y}=2 \mathrm{~mm}$

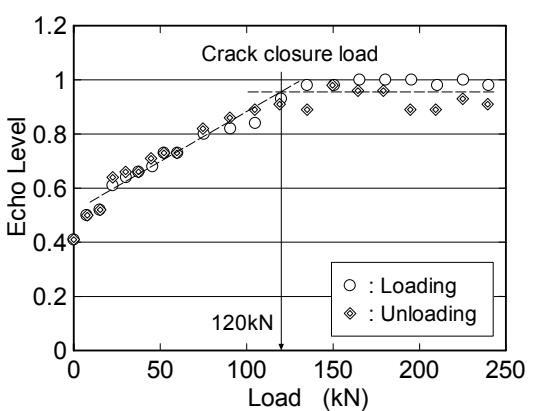

(c) $\mathrm{y}=4 \mathrm{~mm}$

Fig.18 Relationship between load and tip echoes (specimen CC-1 with fatigue crack; depth $=7.5 \mathrm{~mm}$ ).

front, which can be defined as the ratio of the effective stress range $\Delta \sigma_{\text {eff }}$ (a range in stress while a crack tip is open $)$ to the nominal stress range $\Delta \sigma\left(\mathrm{U}=\Delta \sigma_{\text {eff }} /\right.$ $\Delta \sigma)$. This result supports the fact that the crack propagated not in semielliptical shape but in semicircular shape as shown in Fig.14(a). According to a stress intensity factor equation proposed by Newman and Raju ${ }^{13)}$, assuming a semicircular surface crack in a plate with no crack closure along the tip, the stress intensity factor at the crack tip on a surface (a point $\mathrm{C}$ in Fig.13) is larger than that at the deepest point (a point A in Fig.13). In other words, the crack tip at the point $\mathrm{C}$ propagates faster than that at the point $\mathrm{A}$. Therefore, the crack forms semielliptical shape without the crack tip closure, as discussed in below.

Fig.19 shows the measured tip echoes in specimen CC-2 when the crack reached $5.5 \mathrm{~mm}$ in depth. The probe locations are $\mathrm{y}=-2 \mathrm{~mm}, 0 \mathrm{~mm}$ and $2 \mathrm{~mm}$. No change in magnitude of tip echoes was detected in the process of loading and unloading. It means that the high tensile residual stresses introduced by welding discouraged the crack tip against closing, resulting in the semielliptical crack shape as shown in Fig.14(b).

The measurements for a $14 \mathrm{~mm}$ deep crack in specimen CC-2 at locations of $\mathrm{y}=-2 \mathrm{~mm}, 0 \mathrm{~mm}$ and $2 \mathrm{~mm}$ are shown in Fig.20. Even though the crack propagated and entered the area where compressive residual stresses had originally existed (the boundary between tensile and compressive residual stress in the initial condition was around $8 \mathrm{~mm}$ in depth as shown in Fig.15), no closure of the crack tip occurred at the location of $\mathrm{y}=-2 \mathrm{~mm}$ and $0 \mathrm{~mm}$. This behavior is because the residual stress near the fatigue crack tip is tension due to the redistribution of residual stresses with the growth of the fatigue $\mathrm{crack}^{14)}$. The crack tip closure, however, can be observed at the location of $y$ 


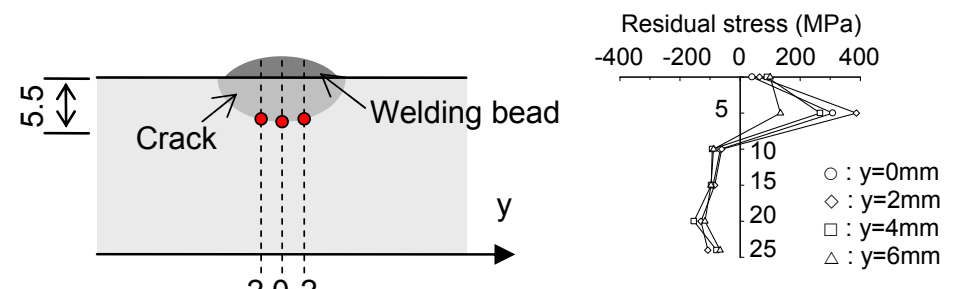

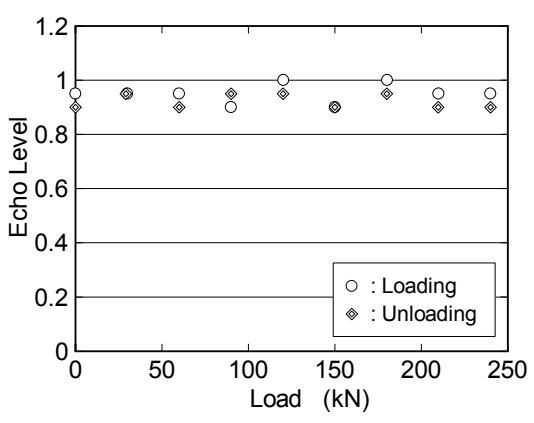

(a) $\mathrm{y}=-2 \mathrm{~mm}$

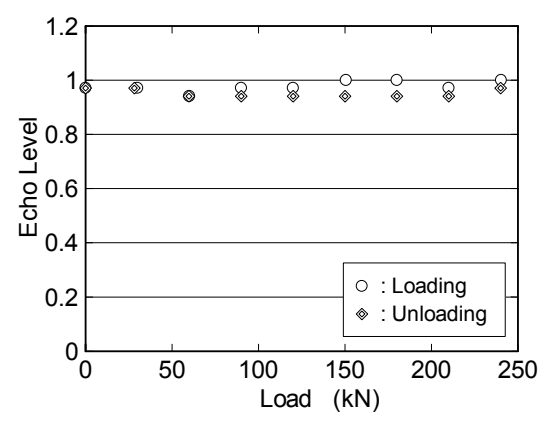

(b) $\mathrm{y}=0 \mathrm{~mm}$

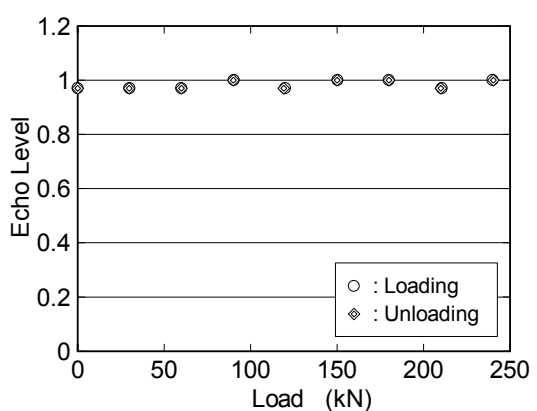

(c) $\mathrm{y}=2 \mathrm{~mm}$

Fig.19 Relationship between load and tip echoes (specimen CC-2 with fatigue crack; depth $=5.5 \mathrm{~mm}$ ).

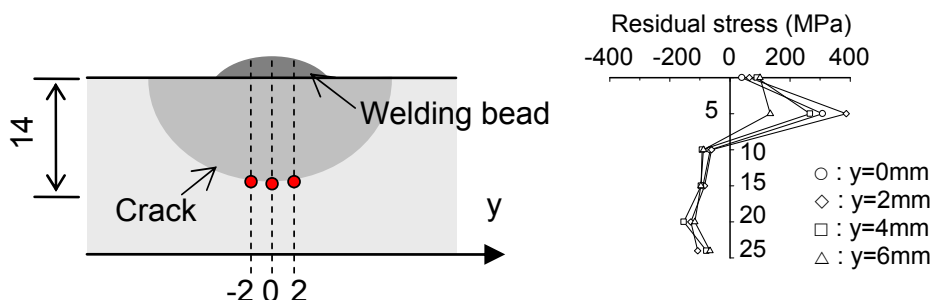

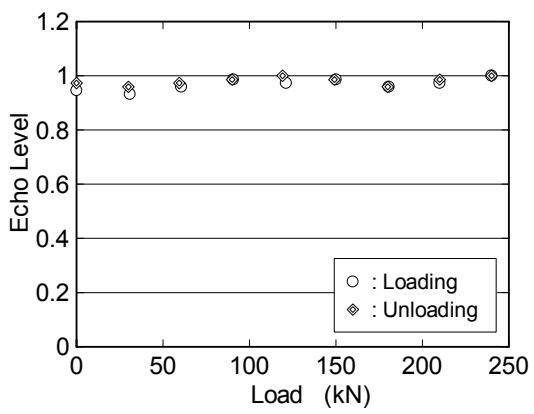

(a) $\mathrm{y}=-2 \mathrm{~mm}$

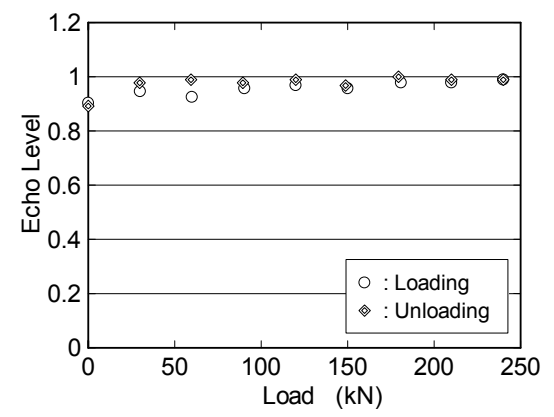

(b) $\mathrm{y}=0 \mathrm{~mm}$

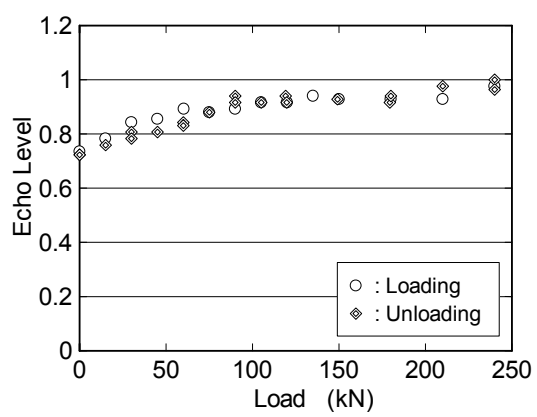

(c) $\mathrm{y}=2 \mathrm{~mm}$

Fig.20 Relationship between load and tip echoes (specimen CC-2 with fatigue crack; depth $=14 \mathrm{~mm}$ ).

$=2 \mathrm{~mm}$, suggesting that the magnitude of the tensile residual stress near the crack tip may be relatively small compared to the other measured points. This result indicates that the residual stress may transit from tension to compression around $14 \mathrm{~mm}$ in depth after the residual stress redistributes by crack propagation. From the measurements, it can be mentioned that the tip echo method has a possibility to detect the fatigue crack closure in welded plates after redistributing and diminishing tensile residual stresses near the tip as crack propagates.

\section{CONCLUSIONS}

The conclusions of this study are listed as follows.
1. It was experimentally observed that distribution of tip echoes with respect to the direction of reflection is non-uniform and that the tip echo in the direction back toward the transmission source is apt to be strong.

2. It was proven that tip echoes show intensity varying with the direction of transmission and that the maximum intensity of tip echoes are obtained when the ultrasonic transmission comes from directly above the slit or crack.

3. The above characteristics of tip echoes are observed regardless of whether the source of tip echoes is an artificially induced slit in the material or a fatigue crack (i.e., regardless of the tip shape).

4. The magnitude of tip echoes from a fatigue crack 
varies with closure and opening of the crack

5. The closure and opening behavior of cracks existing in a plain and a welded steel plate were detected by the tip echo method.

\section{REFERENCES}

1) Bouami, D. and Vadder, D. D. : Detection and measurement of crack closure and opening by an ultrasonic method, Eng. Fract. Mech., Vol. 23, No. 5, pp. 913-920, 1986.

2) Fuji, T., Ootsuka, Y. and Kato, I. : Ultrasonic testing of partial penetration weld, J. JSNDI, Vol. 21, No. 9, pp. 564-565, 1972. (in Japanese)

3) Silk, M. G. : Sizing crack-like defects by ultrasonic means, Research Techniques in Nondestructive Testing, Academic Press, Vol. 3, pp. 51-99, 1977.

4) Harumi, K., Ogura, Y., Miyajima, T. and Uchida, M. : Motion picture of tip waves, J. JSNDI, Vol. 38, No. 9, pp. 849-850, 1989. (in Japanese)

5) Harumi, K. and Uchida, M. : Computer simulation of ultrasonics and its applications, J. NDE, Vol. 9, pp. 81-99, 1990.

6) Miki, C., Fukazawa, M., Katoh, M. and Ohune, H. : Feasibility study on non-destructive methods for fatigue crack detection in steel bridge members, British J. NDT, Vol. 32, No. 6, pp. 291-302, 1990.

7) Harumi, K., Uchida, M., Miyajima, T. and Ogura, Y. : Defect sizing of small inclined cracks on a free surface us- ing multi-tip waves, NDT\&E International, Vol. 25, No. 3, pp. 135-144, 1992.

8) The Japanese Society for Non-Destructive Inspection : Measurement of flaw height using tip echo technique, NDIS 2418, 2005. (in Japanese)

9) Wooh, S.-C. and Shi, Y. : Optimum beam steering of linear phased arrays, Wave Motion, Vol. 29, No. 3, pp. 245-265, 1999.

10) Azar, L., Shi, Y. and Wooh, S.-C. : Beam focusing behavior of linear phased arrays, NDT\&E International, Vol. 33, No. 3, pp. 189-198, 2000.

11) Hirabayashi, M., Miki, C., Tanabe, A. and Shirahata, H. : Ultrasonic testing by the use of plural number of phased array transducers, Doboku Gakkai Ronbunshuu A, Vol. 64, No. 1, pp. 71-81, 2008. (in Japanese)

12) Kikukawa, M., Jono, M., Tanaka, K. and Takatani, M. : Measurement of fatigue crack propagation and crack closure at low stress intensity level by unloading elastic compliance method, J. JSMS, Vol. 25, pp. 899-903, 1976. (in Japanese)

13) Newman, J.C. and Raju, I.S. : Stress-intensity factor equations for cracks in three-dimensional finite bodies subjected to tension and bending loads, NASA Technical Memorandum 85793, 1984.

14) Miki, C., Nishino, F., Hirabayashi, Y. and Takena, K. : Influence of residual welding stress on fatigue crack growth rate, Proc. of JSCE, No. 330, pp.161-168, 1983.

(Received October 15, 2009) 